Introduction: Positron emission tomography/computed tomography (PET-CT) is one of the modalities that allow for imaging the metabolism of human cells. One of the indications for PET-CT is head and neck cancer staging and restaging.

Aim of study: To test the diagnostic performance of PET-CT in a single centre study for head and neck cancer patients.

Materials and methods: The study involved 60 patients with head and neck cancer diagnosed and treated in our department during 2008-2010. There were 71 PET-CT examinations (in 11 patients multiple studies were performed). In 40 patients PET-CT was performed for pre-operative staging, mainly to assess the presence of lymph node metastases. In 23 cases PET-CT was performed to confirm clinical suspicion of tumour recurrence. In 8 patients, the examination was performed to find the origin of the unknown primary cancer. Results: Positron emission tomography/computed tomography results were compared with conventional imaging modalities and histopathology. In the surgical treatment group 4 false positive findings after histological examination were detected. PET-CT in 2 patients failed to show metastasis to the regional lymph nodes (false negative report). In patients with suspected recurrent tumour the diagnosis was confirmed in 14 cases. In all patients with metastases from an unknown primary origin, PET-CT was positive ( 4 cases were confirmed clinically). The results of PET-CT studies helped to change the method of treatment in 21 cases.

Conclusion: Positron emission tomography/computed tomography may be a valuable tool in detecting, staging and monitoring of selected patients with head and neck cancer, with a significant impact on clinical decision making.

Key words: cancer diagnosis, head and neck cancer, PET-CT.

\section{Positron emission tomography in diagnosis and follow-up of treatment of head and neck tumours. Our initial experience}

\author{
Tomasz Piętka ${ }^{1}$, Mirosław Dziuk ${ }^{12}$, Grzegorz Krzymański ${ }^{1}$
}

1Department of Maxillofacial Surgery, Military Institute of Medicine, Warsaw ${ }^{2}$ Mazovian PET-CT Centre, Warsaw

Positron emission tomography/computed tomography (PET-CT) is a modern test. Indications to perform it are expanding. The test allows for imaging of metabolic activity of human tissues [1].

Special diagnostic value of PET-CT derives from the theoretical possibility of imaging many physiological processes in the human body. The test uses chemical compounds which are absorbed and metabolized by human tissue [1]. Positron emission tomography/computed tomography is used in cancer diagnosis, neurology and cardiology [2]. Cancer diagnosis primarily makes use of the phenomenon of increased glucose metabolism in neoplastic cells, known since the 1930s (Warburg et al.) [3].

The test involves intravenous administration of glucose marked by the radioactive isotope of fluorine ${ }^{18} \mathrm{~F}$ (18-FDG, fluorodeoxyglucose). After the time necessary for the preparation's redistribution to tissues, positron emission tomography is performed to depict the locations of increased accumulation of the marker [4]. Revealed foci may suggest the presence of a tumour which is developing fast and is metastatic $[1,5]$. Combining the test with classic images of digital computed tomography allows for precise anatomical localization of a suspected focus [6-8]. Test results are interpreted by a specialist in nuclear medicine and clinicians. Maximum standardized uptake value (SUV) of the radioactively marked metabolite is assessed and compared to physiological uptake. Positron emission tomography/computed tomography imaging is correlated with clinical data.

In worldwide literature this method's sensitivity is assessed as $71-100 \%$ [7-9] and specificity 75-98\% [8-10]. Positron emission tomography/computed tomography system resolution is approximately $3 \mathrm{~mm}$. The low number of reports in Polish literature connected with the use of PET-CT in oral and maxillofacial oncology as well as access to the latest test convinced us to present our initial observations.

\section{Materials and methods}

From January 2008 to June 2010 the PET-CT test was performed in 60 patients with diagnosed cancer of the head and neck region. Patients' age ranged from 28 to 86 (average 63 years). In total 71 PET-CT tests were performed (in some patients the test was repeated during oncological observation).

In 40 cases the test was performed to determine the stage prior to surgical treatment. In 8 patients PET-CT was performed to detect the probable primary origin of cancer. Twenty-three PET-CT tests were performed for sus- 
pected loco-regional tumour recurrence after finishing the treatment (the test was repeated during clinical observation in 11 patients).

In the course of preparation for the procedure all the patients did not eat for about 4 hours. Before the test the patients were given $1000 \mathrm{ml}$ of drinking water. Next, intravenous injection of glucose marked with ${ }^{18} \mathrm{~F}$ isotope was performed (FDG-PET-CT).

Marker dosage was determined individually, depending on body mass. Radioactivity of glucose preparations at the moment of intravenous administration ranged from 272 to $448 \mathrm{mBq}$ (mean $338 \mathrm{mBq}$ ). PET-CT tests were performed within 40-110 min (approximately $60 \mathrm{~min}$ ) after ${ }^{18} \mathrm{~F}-\mathrm{FDG}$ administration. By the use of positron tomography foci of increased accumulation of fluorodeoxyglucose were scanned (fig. 1). Lymph nodes of SUV over 2 or focal asymmetric uptake greater than background were interpreted as a suspected growth process. Test result analysis considered current clinical manifestations and accessory tests (ultrasonography, CT, histopathological tests). Test results are presented in the form of both printouts which show the distribution of metabolic activity within the head and neck and interpretations (fig. 2).

\section{Results}

Altogether, 71 PET-CT tests were performed. A focus of increased metabolism was observed in 56 cases. In 15 tests ${ }^{18}$ F-FDG uptake was within physiological norms in locations of suspected cancer.

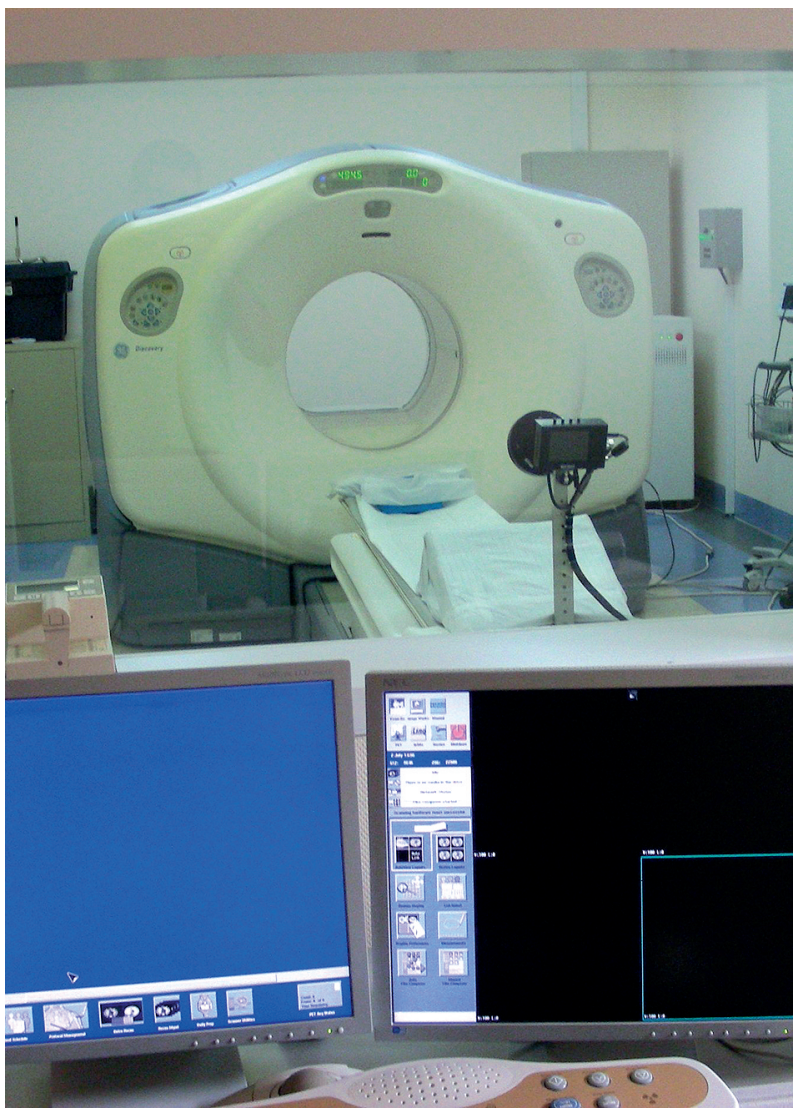

Fig. 1. PET-CT machine
Metabolic activity of the location considered as the primary origin ranged from 3 to 17 SUV (mean 6.8 SUV). Within the neck region the uptake ranged from 1.7 to 13.3 SUV (mean 4.2 SUV).

In 40 patients the test was performed to determine the extent of the cancerous process. In 22 cases in this group the PET-CT result confirmed the previous assessment of clinical stage established based on imaging diagnostics (USG, $\mathrm{CT}$ ) and the scope of the planned procedure. In 8 cases the test revealed foci of increased metabolism located outside the region of planned tissue resection. Therefore, it was necessary to extend the surgery to lower lymph nodes or ipsilateral lymph nodes. In 6 cases the scope of the procedure was limited due to the lack of metabolic activity in the neck region and the lack of clinical signs of metastases to lymph nodes. In 4 patients considerable loco-regional staging of the neoplastic process was revealed (invasion of carotid vessels, region of lung apex and prevertebral muscles). Therefore, these patients were disqualified from surgical treatment and referred for palliative treatment.

Analysis of postoperative histopathology test results showed that in 4 cases metastases to lymph nodes of the neck were not confirmed despite the fact that they showed increased metabolism in PET-CT (false positive reports). In these cases an inflammatory process was determined as the reason for increased uptake. In 2 patients the test did not reveal micro-metastatic foci in lymph nodes in the direct neighbourhood of the primary origin (false negative report).

In 23 patients PET-CT was performed following the end of the treatment within medical check-up (suspected cancer recurrence, fig. 3). In 14 patients in this group regions of increased metabolic activity which indicated tumour progression were observed. In 4 patients the suspected regions were observed within the chest, in 8 patients within lymph nodes of the neck, and in 2 cases within the root of the tongue. In 2 patients another operation was performed. The other patients, following oncological consultation, were referred for palliative treatment.

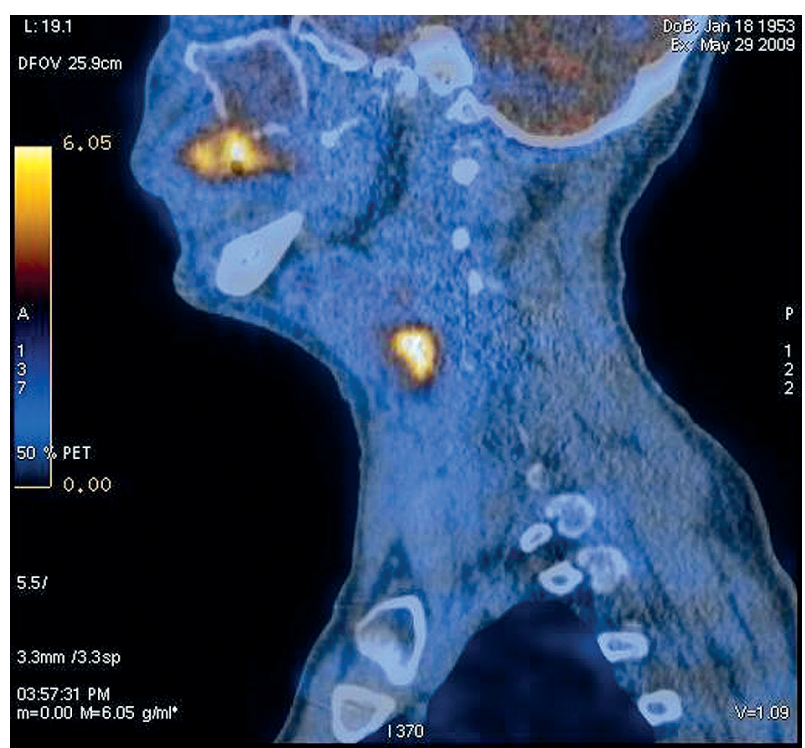

Fig. 2. PET-CT examination. Increased metabolic activity in lower jaw tumour and upper cervical lymph nodes 


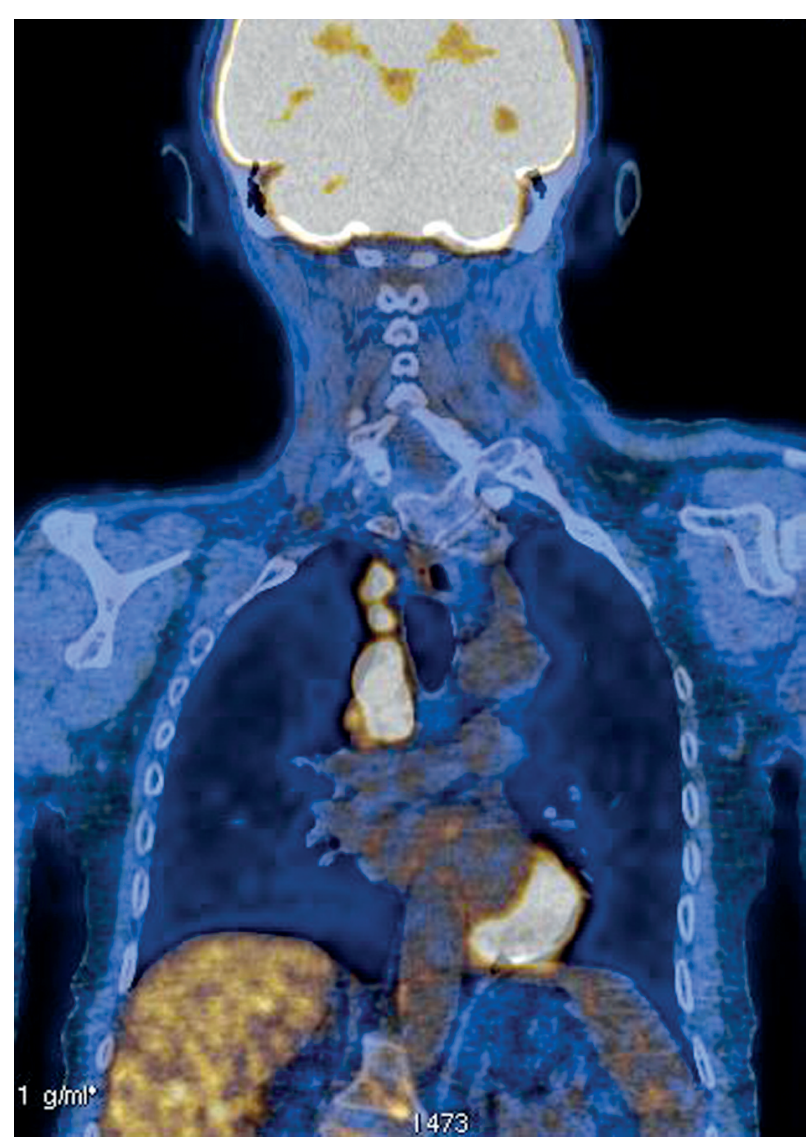

Fig. 3. PET-CT control study. Metastatic squamous cell carcinoma of floor of mouth and the mediastinal lymph nodes

In the group of 8 patients examined for the primary origin of the cancer, in 3 cases regions of increased metabolic activity within the palatine tonsils were observed (fig. 4). Tonsillectomy was performed on 2 patients. In 1 case presence of tonsil cancer was confirmed while in the other case local inflammation was observed. PET-CT in the third patient showed considerable loco-regional staging of cancer (numerous metastases not revealed previously). Following oncological consultation the patient was referred for chemoradiotherapy.

\section{Discussion}

The FDG-PET-CT test allows for assessment of potential staging of neoplastic disease. It also provides the possibility of more precise decisions regarding the scope of the procedure. It is especially significant in older patients with systemic symptoms. The short duration of the procedure, greater precision, decreased loss of blood as well as decreased anaesthesia and analgesics administration have a significant role in peri- and post-surgical prognosis.

Diagnostic possibilities of each test are limited. PET-CT does not allow for imaging of all the potential cancer foci. Micro-metastases smaller than $5 \mathrm{~mm}$ which show considerably small need for glucose (low radioactivity) are not revealed in the test [11-13]. This was also confirmed by our initial observations.

Due to its high sensitivity, PET-CT reveals potential foci both cancerous and inflammatory which show increased

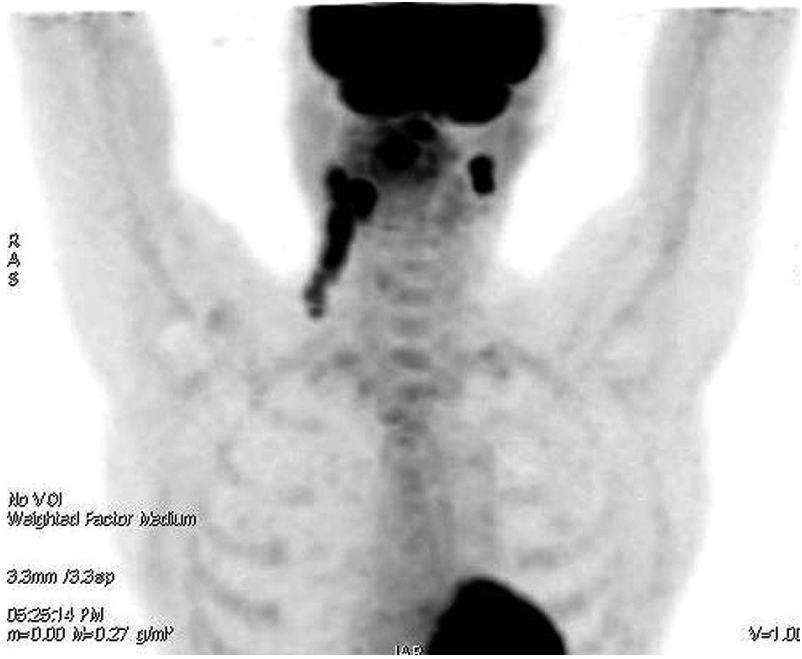

Fig. 4. PET-CT study performed to determine the primary origin of a tumour

glucose metabolism. Therefore, test results require thorough interpretation by a team of experienced clinicians [12].

\section{In conclusions:}

1. The PET-CT test allows for pre-surgical assessment of cancer stage and might be useful in deciding on the scope of surgery.

2. In the case of unknown-origin metastases to lymph nodes it provides new opportunities to search for the primary cancer origin.

3. Positron emission tomography/computed tomography provides an opportunity to monitor neoplastic disease post-surgically.

\section{References}

1. Wong WL, Batty V, Role of PET/CT in maxillo-facial surgery. Br J Oral Maxillofac Surgery 2009; 47: 259-67.

2. Wood KA, Hoskin PJ, Saunders MI. Positron emission tomography in oncology: a review. Clin Oncol 2007; 19: 237-55.

3. Pedersen PL. Warburg, me and Hexokinase 2: Multiple discoveries of key molecular events underlying one of cancers' most common phenotypes, the "Warburg Effect", i.e., elevated glycolysis in the presence of oxygen. J Bioenerg Biomembr 2007; 39: 211-22.

4. Hain FS. Positron emission tomography in cancer of head and neck. Br J Oral Maxillofac Surgery 2005; 43: 1-6.

5. Tamara L, Valez I, Tamara C. Positron emission tomography: a promising diagnostic sodality for head and neck pathology. J Oral Maxillofac Surg 2006; 64: 1272-7.

6. Gordin A, Golz A, Keidar Z, Daitzchman M, Bar-Shalom R. The role of FDG-PET/CT imaging in head and neck malignant conditions: impact on diagnostic accuracy and patient care. Otolaryngol Head Neck Surg 2007; 137: 130-7.

7. Poeppela TD, Krauseb BJ, Heusnerc TA, Boya C, Bockischa A, Antochc G. PET/CT for the staging and follow-up of patients with malignancies, Eur J Radiol 2009; 70: 382-92.

8. Syed R, Bomanji JB, Nagabhushan N, Hughes S, Kayani I, Groves A, Gacinovic S, Hydes N, Visvikis D, Copland C. Impact of combined 18F-FDG PET/CT in head and neck tumours. Br J Cancer 2005; 92: 1046-50.

9. Vogel WV, Wensing BM, van Dalen JA, Krabbe PF, van den Hoogen FJ. Optimised PET reconstruction of the head and neck area: improved diagnostic accuracy, Eur I Nucl Med Mol Imaging 2005; 32: $1276-82$ 
10. Ekberg T, Soerensen J, Engstroem M, Blomquist E, Sundin A, Anniko M. Clinical impast of positron emission tomography (PET) with (18F) fluorodeoxyglucose (FDG) in head and neck tumours, Acta Otolaryngol 2007; 127: 186-93.

11. Fischer MB, Olsen WB, Ley CD, Klausen TL, Mortensen J, Hojgaard L, Kristjansen P. How few cancer cells can be detected by positron emission tomography? A frequent question addressed by an in vitro study. Eur J Nucl Med Mol Imaging 2006; 33: 697-702.

12. Hojgaard L, Specht L. PET/CT in head and neck cancer. Eur J Nucl Med Mol Imaging 2007; 34: 1329-33.

13. Kim SY, Roh JL, Kim JS, Ryu CH, Lee JH, Cho KJ, Choi SH, Nam SY. Utility of FDG PET in patients with squamous cell carcinomas of the oral cavity. Eur J Surg Oncol 2008; 34: 208-15.

\section{Address for correspondence}

\section{Tomasz Piętka}

Department of Maxillofacial Surgery

Military Institute of Medicine

ul. Szaserów 128

04-141 Warszawa

e-mail: stomek@poczta.onet.pl 